\title{
Frontières
}

\section{Abbas Kiarostami ou l'épreuve de la finitude}

\section{Julien Milly}

Volume 19, numéro 2, printemps 2007

\section{Penser sa mort?}

URI : https://id.erudit.org/iderudit/017497ar

DOI : https://doi.org/10.7202/017497ar

Aller au sommaire du numéro

Éditeur(s)

Université du Québec à Montréal

ISSN

1180-3479 (imprimé)

1916-0976 (numérique)

Découvrir la revue

Citer cet article

Milly, J. (2007). Abbas Kiarostami ou l'épreuve de la finitude. Frontières, 19(2), 47-52. https://doi.org/10.7202/017497ar

\section{Résumé de l'article}

L'oeuvre de Kiarostami, entièrement travaillée par les événements du seuil, interroge la figuration des marges. Le vent nous emportera et Le goût de la cerise retracent les moments liés au funeste, ce temps improbable aux confins de la vie crépusculaire. Le réalisateur, à chaque fois, pense une scénographie funeste : dévitalisation de la scène filmique et investissement des zones frontalières, valorisation de formes éphémères ou dégradées ainsi que d’une poétique miroitante.
Ce document est protégé par la loi sur le droit d'auteur. L'utilisation des services d’Érudit (y compris la reproduction) est assujettie à sa politique d'utilisation que vous pouvez consulter en ligne.

https://apropos.erudit.org/fr/usagers/politique-dutilisation/ 


\section{Résumé}

L'œuvre de Kiarostami, entièrement travaillée par les événements du seuil, interroge la figuration des marges. Le vent nous emportera et Le goût de la cerise retracent les moments liés au funeste, ce temps improbable aux confins de la vie crépusculaire. Le réalisateur, à chaque fois, pense une scénographie funeste: dévitalisation de la scène filmique et investissement des zones frontalières, valorisation de formes éphémères ou dégradées ainsi que d'une poétique miroitante.

Mots clés: scénographie du non-lieu irreprésentable - miroir - vitaliser.

\section{Abstract}

Shaped entirely by threshold events, Kiarostami's oeuvre challenges how the margins are represented. The Wind Will Carry Us and A Taste of Cherry are accounts of moments linked to death, the improbable time at the far reaches of the twilight of life. He designs each film to evoke mortality; he creates lifeless onscreen images, pushes into boundary areas, and highlights forms that are ephemeral, faded and shimmeringly poetic.

Keywords: Design for a non-place Unrepresentable - Mirror - Vitalizing

\section{ABBAS KIAROSTAMI OU L'ÉPREUVE DE LA FINITUDE}

\begin{abstract}
Julien Milly,
doctorant en études cinématographiques et audiovisuelles, Université de La Sorbonne Nouvelle, France;

chargé de cours en esthétique et en sciences de l'art.
\end{abstract}

\section{Franchir le seuil. Ô premier deuil ${ }^{1}$. Edmond Jabès}

Montrer la mort, représenter l'ignominie d'une chair qui s'étale, se disloque, cerner une ultime expiration, et l'effroi soudain que suscite la vue d'une figure oubliée, abîmée, de telles images soulignent comment l'incommensurable de l'agonie et l'indicible peuvent alimenter la sphère esthétique. Platon, déjà, estimait l'horreur tant elle était susceptible d'engager d'obscurs désirs, lorsqu'à la vue d'un cadavre, Socrate invitait les siens «à se rassasier de ce beau spectacle» (Platon, $439 \mathrm{c}-440 \mathrm{a})$. Est-ce dire que l'abject, ce qui habituellement repousse, soulève le cœur, répugne, mobilise à certains moments une émotion singulière, teintée à la fois d'admiration et de méfiance? «Une chair misérable, vase de dissolution, peut donc devenir belle à ce point, dans les premières heures de la mort? » s'interrogeait également D'Annunzio (1928, p. 133), inquiet d'entendre ce terrible rapprochement entre la grâce et l'affliction.

Abbas Kiarostami, avec Le vent nous emportera et Le goût de la cerise, entend ce funeste-là, hanté par la désolation, traversé d'ombres, comme si la présence tombait sous l'égide d'une mort redoutée. Contrairement à certaines cinématographies de l'agonie - Cris et chuchotements $^{2}$ et Le silence (I. Bergman), Mère et fils (A. Sokourov), Le souffle au cœur (L. Malle) pour en citer quelques-unes - ou à certaines œuvres vidéographiques pensons notamment à Ophélie et les autres (2006) de V. Foloppe où l'artiste transgresse l'irreprésentable des coulisses théâtrales et filme le suicide de personnages féminins ${ }^{3}$, ces « corps fantôme [auxl à l'identité dégradée » (Foloppe, 2006, p. 248) qui rougissent l'autre scène -, les deux films de Kiarostami destituent la figuration morbide. Pourtant, à chaque fois, loin de répudier la finitude, Kiarostami conduit le spectateur à ce qu'il pense sa déchéance, menant à travers le tissu filmique une réflexion sur l'autoportrait, ou encore instaurant une identification entre la place du contemplateur et celle du personnage suicidé.

\section{LA MORT-MIROIR DANS LE VENT NOUS EMPORTERA (1999)}

Assertif, imagé, le titre du film ouvre sur l'indubitable. Le vent nous transporterait donc vers une autre scène, un ailleurs qu'il nous faudrait accepter: le «rectangle de lourde mort sous le ciel noir» qu'imagine, «au bout d'une longue rue », Yves Bonnefoy (1982, p. 133). Si le titre renseigne sur la nature dramatique du film, l'affirmation laconique interroge chez Kiarostami les territoires liminaires. Penser les marges, apercevoir les confins du temps, n'est-ce pas saisir également la nature transitoire de toute vie? "Au corps de l'homme, il ne 
faut donc pas s'arrêter plus que cela, puisqu'il est un passage », écrit Agnès Minazzoli (1996, p. 127). Elle termine: «La porte est ouverte. L'homme se tient sur le seuil. Il est à lui seul ce seuil. Il est le seul à pouvoir le franchir, pourvu qu'il en saisisse l'occasion sans attendre le verdict d'un gardien qui lui dirait: "Trop tard! On ferme" » (Minazzoli, 1996, p. 211). Notre intention est d'expliciter les liens qui unissent ici le lieu filmique et son hors-champ. Nous verrons ainsi comment l'approche de la finitude dans Le vent nous emportera coïncide avec un sourd effondrement, tant sur le plan dramatique que formel. Une oscillation topique estompe en effet les frontières, si bien que l'histoire apparaît constamment décentrée. Enfin, Kiarostami pense notre déchéance à travers une mise en abyme: filmer la mort dans Le vent nous emportera revient à penser une scénographie où l'autoportrait du réalisateur interroge une économie spéculaire funeste.

Un réalisateur et son équipe se dirigent vers Sia Dareh, petit village du Kurdistan iranien situé au cœur de la vallée noire. Là, ils comptent réaliser en secret un documentaire sur la cérémonie funéraire d'une vieille femme. Attendue depuis quelques jours, crainte par les villageois ${ }^{4}$, l'expiration tarde, générant de longues attentes. Du trépas, le spectateur n'aura l'image que de la porte close, infranchissable, et sur laquelle des regards indiscrets se posent chaque jour.

Songer à la mort ici, ne serait-ce pas écarter l'inadmissible, investir le horschamp et construire un en-deçà filmique? Le vent nous emportera célèbre un tel séisme, cet abyme repéré par Jankélévitch qui estimait «le problème de la cessation d'être [...] impensable et [...] scandaleux» (Jankélévitch, 1994, p. 37). Les marges deviennent en conséquence le lieu où s'inscrit l'événement funeste, mais aussi l'espace mélancolique où les personnages sont relégués.

\section{DES FIGURES EN RETRAIT}

\section{UN PARCOURS DE LA DÉSOLATION}

Dès le début, Kiarostami obscurcit le drame. Filmée en plans d'ensemble, généralement en plongée, une voiture arpente les routes en lacets qui sillonnent la campagne iranienne. La mise en scène exalte ainsi les disparitions du véhicule, comme ingéré par les paysages arides. Le spectateur décèle également des voix qui s'entremêlent: un itinéraire à suivre, un arbre isolé, un chemin qui semble ne mener nulle part, mais ignore toujours le motif du voyage.

Les figures restent dans un premier temps exclues du cadre: soit le point de vue révèle l'immensité de collines et de plaines

\section{Abbas Kiarostami}

Cinéaste, né à Téhéran en 1940 et diplômé de la Faculté des beaux-arts. II produit son premier film en 1940. Son œuvre lui a valu une réputation internationale et a été couronnée de nombreux prix.

Le goût de la cerise

Iran, 1998, 35 mm, couleur, 95 min.

Scénario de Abbas Kiarostami

Un homme dans la cinquantaine roule en voiture. Il a décidé de mettre fin à ses jours mais, avant de réaliser son plan, il doit trouver une personne qui lui promettra d'enterrer son corps.

Le vent nous emportera

Iran, 1999, 35 mm, couleur, $118 \mathrm{~min}$.

Scénario de Abbas Kiarostami, d'après un récit de Mahmud Aidin.

Dans un village du Kurdistan, une vieille femme est sur le point de mourir. Un cinéaste installe son équipe de tournage près de sa maison, sous les yeux interrogateurs des villageois. Ces étrangers venus de Téhéran circulent dans le village, se rendent au cimetière pour une meilleure réception des messages par téléphone cellulaire, mais ils n'entreront pas dans la maison où la mort se prépare à faire son œuvre.

abandonnées, soit la caméra adopte le regard des protagonistes sur la campagne. Plusieurs travellings latéraux créent ainsi des abstractions, lorsque les couleurs, jaunes, ocre, vertes, mises en mouvement par la vitesse du véhicule, édifient quelques structures chromatiques filées. Parfois un personnage surgit d'un pré, moissonnant, tandis qu'un autre, qui tient une faux et observe la voiture, annonce la mort. Illustrant l'ultime maturité, ces vues sur le labeur des saisons ne suggèrentelles pas d'autres récoltes? Cicéron, évoquant l'agonie, usait de la métaphore des fruits, «mûrs et cuits au soleil » (Cicéron, 1989, p. 126), qui se détachent. "Cet état, explique-t-il, est pour moi si agréable que, à mesure que j'approche de la mort, je crois voir en quelque sorte la terre et atteindre enfin le port après une longue traversée» (Cicéron, 1989, p. 126). Pareille «décadence», semblable aux élégies du repos chez Bachelard, lorsque «la matière nous attire vers les profondeurs de sa petitesse, à l'intérieur de son grain, jusqu'au principe de ses germes»(Bachelard, 1948, p. 4), s'éprouve au cœur de l'esthétique kiarostamienne : la ligne d'horizon étant localisée en hauteur, à proximité du bord supérieur du cadre, les points de vue célèbrent des formes effondrées.

Puis, un garçon, Behzad, patientant sur un monticule de terre, accueille les voyageurs à un croisement. Il monte dans la voiture, les guide jusqu'au village. Bien que l'enfant connaisse le motif de l'expédition, Farzad, le documentariste, lui demande de se taire. «Ils sont venus chercher un trésor! » est-il prié de répondre aux curieux. Le scénario met donc une nouvelle fois en retrait le spectateur, puisque l'histoire lui est cachée.

\section{DIVAGATIONS}

Arrivés en contrebas du village, Behzad conduit Farzad à travers les ruelles escarpées de Sia Dareh. Le parcours labyrinthique jusqu'au logis, jalonné de passages étroits, de portes rétrécies, d'escaliers inconfortables, souligne la prévalence de zones intermédiaires, qui vante une instabilité spatiale ${ }^{5}$. Cette errance du personnage kiarostamien, saisi à travers les méandres architecturaux, concède au visage une improbabilité surprenante. Le retrait instaure en effet une perte, l'éviction figurative ouvrant un vide, une béance sans mesure, qu'accentue par ailleurs le surcadrage. Les treize premiers plans oblitèrent ainsi l'identité, exclue de la représentation ou éphémère, prête à basculer vers le hors champ, noyée parmi les anfractuosités d'un paysage essoufflé.

La figure de Farzad, longtemps occultée, apparaît au quatorzième plan : au fond d'une étroite ruelle surgit le réalisateur, devancé par le jeune Behzad. Les deux s'approchent de la caméra et, tandis qu'ils franchissent le seuil filmique, un panoramique latéral accompagne leurs marches, de telle manière que l'on puisse observer leurs dos s'éloigner.

Les associés qui accompagnent Farzad resteront en dehors du cadre. Alors qu'ils sont tous logés chez les parents de Malak Khanum, la malade, la mise en scène chante l'incertain. Filmée de manière frontale, la maison reste inaccessible, extérieure. Le dispositif accentue non seulement l'obturation de la profondeur imagée, mais également les trouées architecturales, qui distribuent autant de zones obscures impénétrables. L'intérieur restera en somme virtuel, de même que le réali- 
sateur ne filme pas l'ultime visage. Face au pan de mur, un balcon situe une aire évanescente, en marge de l'irreprésentable, où ne cesse d'apparaître Farzad, transitoire, captif d'un non-lieu scénique, prêt à l'avaler ou à le dissoudre. Cette errance ne raconterait-elle pas, ainsi que l'admet ailleurs Murielle Gagnebin analysant l'œuvre de Giacometti, un chancellement identitaire: "L'étendue contre la figure [...]» (Gagnebin, 2004, p. 188) ? Qu'explicitent en l'occurrence pareils vacillements aux bords de l'image?

\section{FORMES}

\section{DE L'ENGLOUTISSEMENT}

Nombreux sont les auteurs qui ont écrit sur le besoin d'éloigner le corps éteint. Lorsque Georges Bataille, par exemple, cernant les périls qui émanent du funèbre, évoque "cette contagion» (Bataille, 1957, p. 53), Michel de M'Uzan, quant à lui, souligne comment «la crainte ancestrale d'être entraîné, dévoré par le moribond» (de M’Uzan, 1977, p. 193) interpelle une mise à distance. Le vent nous emportera scénographie un tel achoppement.

\section{UNE CIRCULARITÉ FUNESTE}

Trois scènes scandent régulièrement l'histoire: la maison de la grand-mère vue de l'extérieur, l'espace périphérique du balcon, qui, filmé à sept reprises, engage une élégie louant l'instable, et, enfin, le cimetière à la lisière du village, sur les hauteurs. Le vent nous emportera forme ainsi une boucle, des premiers paysages où la voiture lacère les collines cultivées jusqu'au plan final où un os jeté, qui flotte et tourne sur lui-même, est entraîné par un ruisseau. Alors que le retour crée des entrelacs scéniques, l'engloutissement attise une fatale éviction : la figure, noyée, disparaît. «Visages ouverts, visages donnés, mais où il n'y a personne à prendre », écrivait Henri Michaux (1963, p. 41). Sur le plan dramatique, l'absorption identitaire répond à une expansion du thème oral: d'un côté, l'être ingéré par le hors-scène; de l'autre, un ultime repas en offrande à la mourante, livré devant sa porte. L'espace de l'invisibilité suggérant une avidité silencieuse, le mouvement d'extraction figurative appelle un imaginaire nutritif, comme si le sujet, «mort par épuisement» (Kestemberg et al., 1972, p. 220) selon les mots d'Évelyne et Jean Kestemberg, avait alimenté un dehors insatiable.

Le seuil incarnerait ainsi chez Kiarostami les marges funestes. Pierre Fédida, méditant sur la relation à l'absence, émet l'hypothèse d'une incapacité à survivre dans certains cas: " la perte de l'objet (séparation, abandon...) ne comporte une menace que sous cette condition d'entraîner la destruction du moi. [...] l'angoisse de la perte d'objet d'amour se laisse interpréter comme l'angoisse du moi de ne pouvoir survivre au-delà de la disparition de l'objet: la mélancolie est moins la réaction régressive à la perte

Les rochers à Montmajour (1888), Vincent Van Gogh.

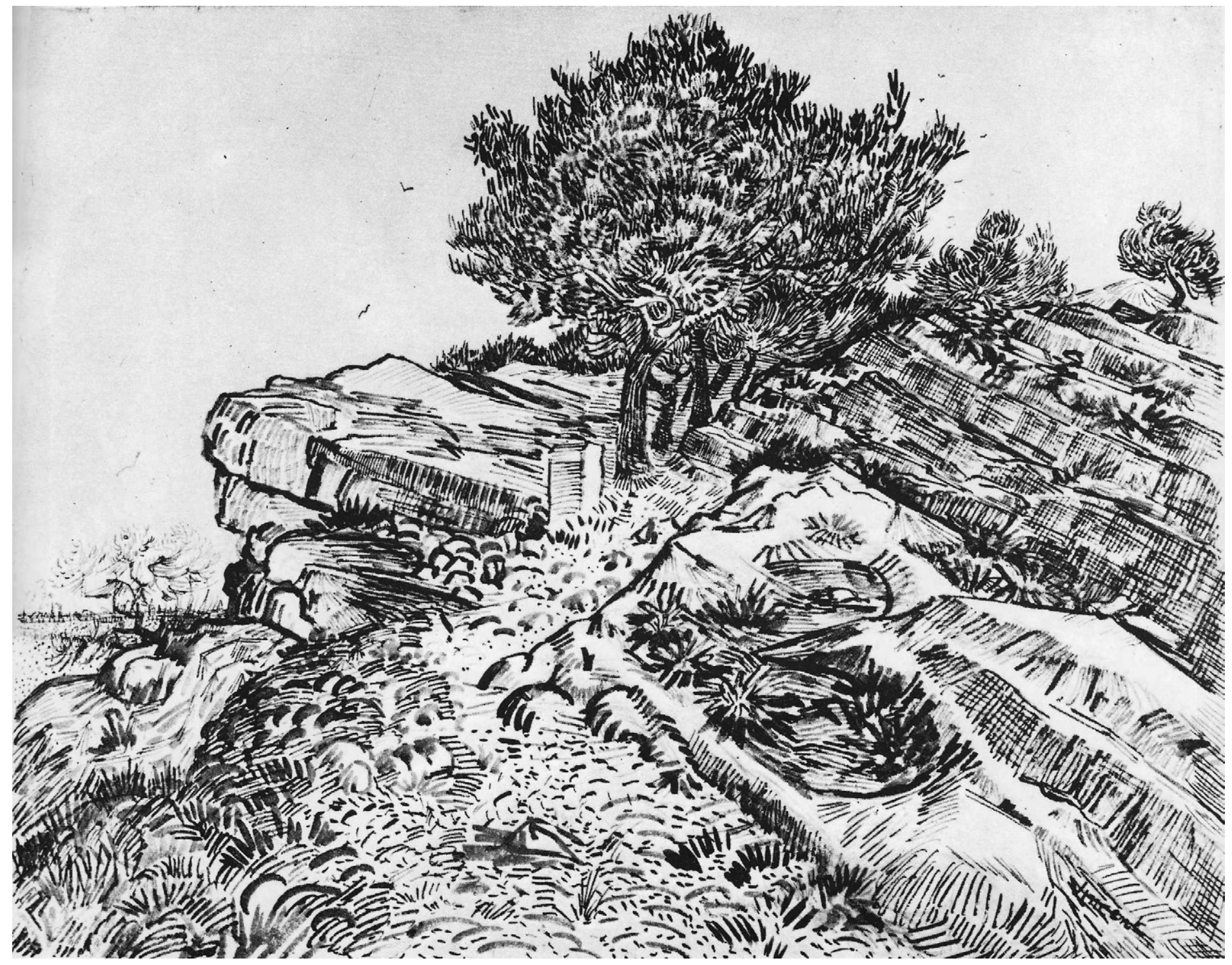


de l'objet que la capacité fantasmatique (ou hallucinatoire) de le maintenir vivant comme objet perdu» (Fédida, 1978, p. 6566). En accord avec Freud, selon qui la mélancolie se traduit entre autres par «la diminution du sentiment d'estime de soi» (Freud, 1968, p. 146-147), Fédida ajoute que le dessèchement identitaire serait un moyen fantasmatique de commercer avec le disparu. L'endeuillé pourrait effacer les conséquences de la rupture, en niant les violences frontalières.

C'est précisément ce que le cinéaste nous invite à examiner : une certaine identification entre l'espace du hors-champ lié à l'événement morbide, et la scène filmique, souvent dévitalisée, anéantie, pulvérisée. Cette mise en continuité qualitative de ces deux versants traduit une fragilisation des bordures, puisque celles-ci tendent à renoncer à leurs vertus limitatives. «Perdre sa peau, écrit Didier Anzieu, c'est perdre les limites du soi, perdre la cohésion des morceaux qui le constituent, perdre le sentiment d'identité »(Anzieu, 1996, p. 4). L'espace chez Kiarostami se désagrège et prolonge les suffocations du hors-champ. La dramaturgie funèbre permettrait en conséquence d'assujettir le visible à la mort annoncée et de reculer le moment où l'intervalle se crée, séparant l'être du nonêtre. Les espaces tendent à se confondre ainsi, l'un s'offrant comme le miroir d'un autre déchu. Ne rejoignons-nous pas ici Jankélévitch, lorsqu'il définit « une limite sans épaisseur»: «bien moins qu'une fine coupure ou une imperceptible couture; entre l'en-deçà et l'au-delà il n'y a pas de zone mixte, mais il n'y a même pas un seuil [...] la mort est l'insécable section de l'Avant et de l'Après [...] un Presque-rien [...] comme un intervalle infinitésimal ${ }^{6}{ }$ ' Le lieu filmique chez Kiarostami s'apparenterait finalement à une marge.

\section{ENSEVELISSEMENTS}

Quatre étapes successives scandent l'enlisement progressif de la figure kiarostamienne. Des effets dissociatifs, pour commencer, mettent en retrait le corps. Le réalisateur expérimente ainsi maintes césures, optant pour des voix off sur de grands paysages silencieux, ou désertés, comme si la parole n'émergeait que d'une autre scène.

Ensuite, certaines vues, marquées par de virulentes plongées, agressent le support identitaire. La caméra, en hauteur, écrase alors la figure et dévoile une «forme déclassée ${ }^{7} »$. Par exemple, c'est l'arrivée des personnages à Sia Dareh, lorsqu'ils escaladent le sentier abrupt; Behzad aperçu du balcon ou encore les riverains au seuil de l'agonie. Ces points de vue insolites oblitèrent la représentation du visage. Ils éclipsent également l'envergure d'une corporéité abattue. Or, selon Fédida, «c'est [...] du modèle de la régression qu'il faudrait attendre une véritable théorie de la déformation [...]»(Fédida, 2000, p. 16).

Troisième procédé qui évince l'intime: l'appétence pour les zones frontalières. Une telle esthétique favorise une économie du transitoire. S'attachant à filmer les passages (balcons, portes, fenêtres, terrasses, ruelles, chemins), le réalisateur vante le basculement de l'être vers le hors-scène. C'est dire que l'espace kiarostamien évoque des états caractéristiques du vacillement. «L'homme habite l'errance», écrit également Jean Duvignaud (1977, p. 133), qui refuse toute dichotomie entre la scène et le non-lieu. Mais n'est-ce pas aussi une spécificité de l'art cinématographique que de mimer le mouvement, la disparition?

Une dernière manière concerne l'ensevelissement. L'espace filmique, qu'il soit creusé ou percé, engloutit la figure, dorénavant enlisée par ces topoï de la dépression. Haut lieu de la mémoire situé à la périphérie du village, le cimetière ponctue régulièrement l'œuvre, lorsque Farzad, recevant un appel téléphonique, est contraint de rejoindre le point culminant des alentours. Il découvre là, sous la terre, au fond d'un gouffre, un homme qui pioche. Non visible, l'ouvrier semble avalé par une profondeur asséchée. Le lieu scénique, métamorphosé en trappe, devient une structure d'élision identitaire. Filmé en plongée, le trou ne laisse apparaître qu'une excavation obscure, un regard de l'ombre.

\section{MOURIR SANS VISAGE}

«Voués à une agonie sans génie, nous ne sommes ni auteurs de nos extrémités, ni arbitres de nos adieux; la fin n'est plus notre fin », écrit Cioran (1949, p. 59), méditant sur la décomposition. Une telle désubjectivation aux termes de la vie instruit une gageure: comment offrir un visage à l'agonie? Chez Kiarostami, le retrait figuratif, loin d'écarter le registre morbide, le déplace tout au contraire. Si le réalisateur nous épargne ainsi l'ultime regard ${ }^{8}$, il forme d'autres entrelacs, qui éclairent et illuminent la déchéance.

\section{UN RETOUR SILENCIEUX}

Kiarostami édifie trois irreprésentables en marge de l'instance filmique: la chambre de la mort, la fosse que l'ouvrier creuse et la cave obscure à l'intérieur de laquelle une jeune femme puise le lait. La laitière retrouve par ailleurs en secret le fossoyeur, avec son récipient, au fond du trou. L'invisibilité fondamentale de ces espaces cryptiques invite à penser un lien significatif entre chacun des événements : l'agonie, l'étreinte amoureuse et un ima- ginaire autour d'une lactation. L'espace de la fin et celui des nourritures primitives tendent ici à se rejoindre. L'épilogue identitaire soulignerait en conséquence, dans Le vent nous emportera, un retour à un érotisme maternel, régression que M. de M'Uzan a mise en évidence lorsqu'il explicite le travail du trépas: "À la veille de leur mort ou dans les heures qui la précèdent, le comportement de certains patients laisse déduire un surprenant élan pulsionnel, une avidité régressive, positivement unheimlich, qui ferait presque parler d'un embrasement du désir» (de M'Uzan, 1977, p. 189). L'auteur poursuit en ces termes : "Grâce à la distension progressive de son être psychique, le mourant absorbe l'objet dans son espace érotique, et si totalement parfois qu'il ne ressent même plus l'absence de la personne réelle [...] Il y a là quelque chose de comparable à l'organisme formé par la mère et son nouveau-né »(de M’Uzan, 1977, p. 196). Une telle appréciation des mouvements confusionnels à l'approche de la mort s'exprime chez Kiarostami par un amenuisement de la scène identitaire : le hors-champ, lieu de la déchéance, absorbe en effet l'événement diégétique, l'engloutissant.

\section{LA MORT DÉVOILÉE}

Bien qu'absente et en marge de la représentation, la figure de l'agonie est longuement mise en abyme par Kiarostami. Diversifiant en effet les regards sur la mourante, le réalisateur médite un dispositif spéculaire habile à distancer et à enclore la décrépitude: il s'agit en première analyse d'un film sur un documentaire qui échoue, l'équipe de tournage n'ayant pas eu la patience d'attendre la fin. Farzad manipulerait en ce sens les intervalles et composerait l'écart ${ }^{9}$. Second intermédiaire à l'insoutenable, l'enfant: médiateur à l'orée du village, il a pour mission d'accompagner les curieux jusqu'au lieu dramatique et de renseigner Farzad sur l'événement morbide. Ce procédé d'encadrement, si l'on suit les travaux de Lucien Dällenbach, constituerait « une manière de passage à la limite»(Dällenbach, 1977, p. 22). La mise en réflexion cinématographique - film dans le film, l'enfant comme figure gémellaire du réalisateur, foisonnements de seuils spéculaires...-, engendre «une espèce d'oscillation $^{10}$ » habile à délocaliser l'intrigue vers les marges. Les incises spéculaires abîment ainsi les lignes de démarcation.

Enfin, la démarche créatrice contourne la difficulté qui consiste à montrer la mort: un dispositif en miroir crée une forme singulière qui, incluant le spectateur, souligne un face-à-face troublant, peut-être cette mort qui dévisage, dont parle longuement Jean-Pierre Vernant ${ }^{11}$. Kiarostami a en 
effet reconstruit au montage une frontalité entre deux balcons: sur l'un, Farzad se rase à l'aide d'un miroir accroché à un mur tandis que, sur l'autre, une femme berce son enfant récemment né. S'il crée une ouverture lumineuse, le pan réflexif renvoie aux commencements, qu'illustre la nouvelle relation dyadique. Un abîme se creuse entre les images: l'homme qui attend la mort et l'enfant qui pleure. "C'est entre les images, rappelle Raymond Bellour, que s'effectuent, de plus en plus, des passages, des contaminations, d'êtres et de régimes [...] Il deviendrait impropre de voir dans l'image quelque chose de sûrement localisable, une entité vraiment nommable» (Bellour, 1999, p. 10). Le seuil permet en l'occurrence de rapprocher des espaces qui s'affrontent, celui de la maternité et celui de la mort escomptée. L'origine est là comme subjuguée par la question de la finitude. Devrions-nous comprendre que toute considération sur les derniers souffles inscrirait un retour aux formes primordiales de la vie? Mais également un retour sur soi, comme le suggère la prégnance de l'autoportrait, lorsque Farzad se rase, l'objectif de la caméra figurant son miroir? Le reporter n'est-il pas en effet une personnification du réalisateur, et le film, $L e$ vent nous emportera, comme une réflexion sur l'être? Or, penser l'autoportrait, selon Montaigne, c'est voir sa figure disparaître: «La mort se mesle et confond par tout à nostre vie: le declin præoccupe son heure et s'ingere au cours de nostre avancement mesme. J'ay des portraits de ma forme de vingt et cinq et de trente cinq ans; je les compare avec celui d'asteure : combien de fois ce n'est plus moy! combien est mon image presente plus esloignée de celles là de celle de mon trespas» (Montaigne, 2004, p. 1102). L'être, une fois de plus, s'envisagerait sur le seuil. Et l'autoportrait du cinéaste interrogerait, en marge de la représentation, le visage agonisant.

\section{DERNIĖRES ERRANCES}

DANS LE GOÛT DE LA CERISE (1997)

Qu'en est-il ainsi chez Kiarostami des formes qui valorisent l'instable, magnifient l'errance jusqu'à souscrire les fondements d'une insaisissable identité? Les visages laissés ou déchus, tremblants parfois, suggèrent-ils ce que Michel de M'Uzan appelle « un flottement du sujet » (de M'Uzan, 1977, p. 162) ? Ou, convoquent-ils l'écho d'un «interminable soliloque avec l'absent» (Fédida, 1978, p. 83), que Pierre Fédida décèle derrière toute figure? Sans doute l'individu est-il soumis à l'art de l'ellipse et nous devrions comprendre, à l'instar d'une histoire de la pensée, que l'être réside sur le passage, siège à l'entre-deux : Jean-François Lyotard (1983) revendiquait ainsi une vie susceptible d'entrouvrir au différend et de creuser maints abîmes. «Éphémère, le corps humain devrait pouvoir élever la pensée à l'idée qu'elle a son lieu dans le changement, la mobilité, la métamorphose [...]», écrit encore Agnès Minazzoli (1996, p. 118), à propos d'une nature humaine qualifiée d'improbable. Des visages furtifs aux présences instables et mouvantes, jusqu'à l'ultime destinée, l'éphémère côtoie ici une esthétique de la traversée.

Le goût de la cerise célèbre de tels séismes. Là où Le vent nous emportera nouait des zones d'obscurités, ce film évacue le personnage central, cherchant à l'anéantir véritablement: un homme d'âge mûr, au volant d'une voiture, alpague quelques promeneurs solitaires à la sortie de Téhéran. Cet homme, Badii, intrigue, et ce d'autant plus que nous ne voyons pas son visage pendant les premières scènes. Alors que des plans subjectifs répétés décrivent l'objet de ses contemplations et de ses rencontres successives, l'errance témoigne d'une incertitude fondamentale: qu'attend-il de ses rapides entrevues? Quelle est la teneur mystérieuse du pacte proposé? Les dialogues, entrecoupés, rendent compte de nouvelles ruptures et chaque opportunité échoue rapidement, de même que les plans sur les territoires desséchés, ou sur les nuages poussiéreux qui s'élèvent, composent des flux dévitalisés. Enfin, après plusieurs longues séquences qui soulignent l'abandon, nous apprenons l'ampleur du marché: Badii est à la recherche d'un compagnon qui, en contrepartie d'une somme d'argent, accepterait de l'ensevelir.

Filmant à de multiples reprises les disparitions de son personnage, Kiarostami va également dissocier, lors d'une scène qui se déroule à la cimenterie, l'acteur de son ombre. Arrivé au fond de ce lieu encaissé au milieu de monticules terreux, Badii sort de son véhicule. Il marche le long d'une large tranchée, s'arrête et regarde la profondeur de l'anfractuosité. Son ombre se reflète à présent de l'autre côté du sillon, sur la tranche sableuse, transformée ici en un véritable écran second. L'image de son corps, projetée sur une matière friable et qui menace de s'écrouler, est ensuite recouverte par une coulée de terre qui saillit du haut. De la sorte, l'ombre identitaire se désagrège parmi les fines particules de la cimenterie et, lorsque le cadre se resserre sur le reflet obscur de l'acteur, le tragique est comme redoublé: même cette image du corps est anéantie, agressée un instant, absorbée par l'abîme. Cette configuration réflexive interpelle une iconographie de type narcissique où la mort du double annonce in fine l'exténuation du personnage central. Or, chez Kiarostami, celui-ci apparaît comme l'ombre du contemplateur!
La séquence terminale magnifie enfin les mécanismes de la traversée. Ici, l'acteur du film disparaît, laissant place à de nouvelles perspectives indécises, et qui appellent l'imaginaire toujours versatile du spectateur ${ }^{12}$.

Badii a quitté Bagheri, l'homme qui a accepté de l'accompagner jusqu'à la mort. Le rendez-vous est fixé, au pied d'un arbre, en contrebas d'une chaussée, au milieu des collines. Un plan large de nuit découvre le mouvement circulaire d'une voiture. Un taxi dépose un homme: assis de dos, sur la lisière du chemin, Badii contemple la nuit céleste, puis s'engouffre par la partie inférieure du cadre. L'image vidée est suivie d'un second plan où l'on voit, en plongée verticale, son visage, les yeux ouverts sur un ciel chargé. Puis, un plan subjectif illustre la vue du fond de la fosse: le vent souffle d'épais nuages grisâtres sur la lune; un orage déchire violemment l'espace cotonneux. À la mesure des sourdes détonations, le visage s'enlève plusieurs fois jusqu'à ce que l'écran devienne entièrement noir. Le bruit d'une averse figure les menaces d'un ensevelissement et commémore l'épisode de la cimenterie. En épousant le point de vue de l'homme prêt à mourir, la caméra n'invite-t-elle pas le spectateur à considérer son propre suicide? L'obscurité, dans cette perspective, figure un sourd étouffement, qui entraîne l'acteur-spectateur jusqu'au désêtre.

Enfin, alors qu'on entend un crescendo de voix sans pouvoir distinguer les quelques paroles prononcées, l'image dévoile progressivement un paysage ensoleillé où l'on reconnaît rapidement l'arbre, les routes en lacets. L'image, tournée pour la fin du film en vidéo, diffère cependant: rugueuse, prête à se disloquer, elle témoigne d'une nouvelle précarité. Le grain s'est coloré de tonalités plus franches et la lumière baigne la scène tout en la surexposant légèrement. $\mathrm{Au}$ loin, de jeunes militaires, qui effectuent une course à pieds, chantonnent. La longue ellipse temporelle amène le spectateur à s'interroger sur le sort de Badii : Bagheri est-il venu l'enterrer? Filmée de manière à ce que l'image vacille, la scène montre à présent un caméraman, suivi d'un technicien du son. Après un panoramique, Badii surgit, une cigarette à la main. Un homme s'écrie au loin: "Dites aux hommes de s'arrêter, le tournage est terminé... on vient juste pour la prise de son. » L'acteur Badii remonte dans sa voiture, démarre puis expire au détour d'un ultime virage. L'écran noir achève l'œuvre. La mise en abyme cinématographique accentue la dimension terminale: si le personnage a disparu, laissant l'œuvre ouverte, l'acteur lui-même n'est plus qu'un être improbable, et qui s'est consumé à l'image de la 
cigarette qu'il tenait. En quittant la scène du tournage, celui-ci devient comme une image du spectateur, invité lui-même à éprouver une fin dramatique.

Qui donc nous a retournés

de la sorte que,

quoi que nous fassions,

nous avons toujours l'air

de celui qui s'en va ${ }^{13}$ ?

R. M. Rilke

Le transitoire caractérise ainsi l'espace fondamental du deuil, lorsque personnages et acteurs sont absorbés vers un hors-cadre funeste. Avec Kiarostami, la mort interroge non seulement les formes de l'errance le dernier portrait -, mais également la forme filmique elle-même qui tend à se dissoudre. Les vers de Rilke, quant à eux, montrent comment filmer sa propre mort reviendrait en définitive à exalter les formes du passage.

\section{Bibliographie}

ANZIEU, D. (1996). Les enveloppes psychiques, Paris, Éditions Dunod.

BACHELARD, G. (1948). La terre et les rêveries du repos. Essai sur les images de l'intimité, Paris, Librairie José Corti.

BATAILLE, G. (1957). L'érotisme, Paris, Les Éditions de Minuit.

BELLOUR, R. (1999). L'entre-Images 2. Mots, images, Paris, P.O.L. éditeur, coll. "Trafic».

BELLOUR, R. (dir.) (1990). Passages de l'image, Paris, Éditions du Centre Pompidou.

BERGALA, A., «L'intervalle » dans AUMONT, J. (dir.) (2000). La mise en scène, Bruxelles, Éditions De Boeck Université.

BONNEFOY, Y. (1982). «Hier régnant désert » dans Poèmes, Paris, Éditions Gallimard.

CICÉRON (1989), Caton l'Ancien. De la vieillesse, Paris, Les Belles Lettres, coll. «Universités de France».

CIORAN, E. (1949), Précis de décomposition, Paris, Éditions Gallimard, coll. «Tel».

DÄLLENBACH, L. (1977). Le récit spéculaire. Essai sur la mise en abyme, Paris, Éditions du Seuil, coll. «Poétique».

D'ANNUNZIO, G. (1928). Contemplation de la mort, Paris, Calmann-Lévy.

DE M'UZAN, M. (1977). De l'art à la mort, Paris, Éditions Gallimard, coll. «Tel».

DIDI-HUBERMAN, G. (1995). La ressemblance informe ou le gai savoir visuel selon Georges Bataille, Paris, Éditions Macula.

DUVIGNAUX, J. (1977). Lieux et non-lieux, Paris, Éditions Galilée.

FÉDIDA, P. (2000). Par où commence le corps humain. Retour sur la régression, Paris, PUF, coll. «Petite bibliothèque de psychanalyse ».

FÉDIDA, P. (1978). L'absence, Paris, Éditions Gallimard.
FOLOPPE, V. (2006). « Rougir l'autre scène» dans GAGNEBIN, M. et J. MILLY (dir.), Les images honteuses, Seyssel, Éditions Champ Vallon, coll. "L'Or d'Atalante», p. 238-249.

FREUD, S. (1968). «Deuil et mélancolie» dans Métapsychologie, Paris, Éditions Gallimard.

GAGNEBIN, M. (2004). Authenticité du faux. Lectures psychanalytiques, Paris, PUF, coll. «Le Fil rouge».

HÉRAN, E. (dir.) (2002). Le dernier portrait, Paris, Éditions de la Réunion des musées nationaux.

JABÈS E. (1990). Le seuil. Le sable. Poésie complète 1943-1988, Paris, Éditions Poésie / Gallimard.

JANKÉLÉVITCH, V. (1994). Penser la mort, Paris, Éditions Liana Levi.

JANKÉLÉVITCH, V. (1977). La mort, Paris, Éditions Flammarion, coll. "Champs».

KESTEMBERG, É., J. KESTEMBERG et S. DECOBERT (1972). La faim et le corps. Une étude psychanalytique de l'anorexie mentale, Paris, PUF, coll. «Le Fil rouge».

KIAROSTAMI, A. (2002). Le vent nous emportera, Paris, Cahiers du Cinéma, coll. «Petite Bibliothèque des Cahiers du Cinéma».

LIANDRAT-GUIGUES, S. (2005). Esthétique du mouvement cinématographique, Paris, Éditions Klincksieck, coll. «50 questions».

LYOTARD, J.-F. (1983). Le différend, Paris, Les Editions de Minuit, coll. "Critique».

MICHAUX, H. (1963). Passages, Paris, Éditions Gallimard, coll. "L'imaginaire».

MILLY, J., «L'orée de l'agonie: Cris et chuchotements » dans JIMENEZ, M. (dir.) (2003). Imaginaire et Utopie du XXI $I^{e}$ siècle, Paris, Éditions Klincksieck.

MINAZZOLI, A. (1996). L'homme sans image. Une anthropologie négative, Paris, PUF, coll. "Perspectives critiques».

MONTAIGNE, M. (de) (2004). Les Essais, Paris, PUF, coll. "Quadrige».

RILKE, R. M. (1972). Poésie. Euvres II, Paris, Éditions du Seuil.

VERNANT, J.-P. (1998). La mort dans les yeux. Figures de l'Autre en Grèce ancienne, Paris, Hachette Littératures, coll. «Pluriel».

\section{Notes}

1. E. Jabès, Le seuil. Le sable. Poésies complètes 1943-1988, Paris, Éditions Poésie / Gallimard, 1990, p. 11.

2. Voir à ce sujet notre étude: «L'orée de l'agonie. Le non-lieu dans Cris et chuchotements » dans M. Jimenez (dir.), Imaginaire et Utopie $d u X X I^{e}$ siècle, Paris, Éditions Klincksieck, 2003, p. 109-122.

3. Installation exposée notamment à la Troisième Biennale d'Art contemporain de Bourges (France), du 9 au 19 novembre 2006. Avec Lady M. s'en lave les mains, la figuration de la mort passe par une anamorphose du visage épris, véritable larme rougeoyante qui efface un dernier regard éploré.
4. Les premières esquisses du film diffèrent de l'histoire définitive: «Dans l'idée originale du film, le documentariste, pour parvenir à son but, se voit obligé de négocier sa mort avec la malade. Celle-ci acceptait de feindre la mort pour que le projet se réalise et pour que l'équipe puisse tourner le film [...] Au chevet de cette vieille femme au seuil de la mort, nous avons compris combien il était difficile de rester fidèle à l'idée d'origine. » (A. Kiarostami, « Préface», dans Le vent nous emportera, Paris, Cahiers du cinéma, coll. Petite bibliothèque des Cahiers du cinéma », 2002, p. 5.)

5. Voir R. Bellour, C. David, C van Assche, "Préface», dans Passages de l'image, Paris, éditions de Centre Pompidou, 1990, p. 7. "Comme si c'était là, dans cet étagement, cet enlacement de passages, que l'image trouve aujourd'hui son lieu, sa plus entière et nouvelle qualité d'énigme, dans une infinité suspendue de mouvements et de temps $[\ldots] »$.

6. V. Jankélévitch, La mort, Paris, Éditions Flammarion, coll. «Champs», 1977, p. 270-271. L'auteur souligne par ailleurs comment toute philosophie du seuil reste en marge de son objet: «[...] philosophant autour d'un presque-rien beaucoup trop fin pour être connu, elle survient soit l'instant d'avant [...] soit l'instant d'après [...] En avance, en retard!» p. 38.

7. G. Didi-Huberman, La ressemblance informe ou le gai savoir visuel selon Georges Bataille, Paris, Éditions Macula, 1995, p. 135.

8. Il faudrait donc ajouter ici à la tradition du dernier portrait la perspective kiarostamienne: le regard des marges. Voir sur la question: E. Héran (dir.), Le dernier portrait, Paris, Éditions de la Réunion des musées nationaux, 2002.

9. Voir à ce sujet A. Bergala, "L'intervalle», dans J. Aumont (dir.), La mise en scène, Bruxelles, Éditions De Boeck Université, 2000, p. 34.

10. Ibid. p. 47. À propos du rapport spéculaire, Dällenbach note comment le principe d'identité spatiale vacille: «C'est là dire que le miroir-espion a moins à charge ici d'intégrer au roman une réalité "extérieure" que d'abolir l'antithèse du dedans et du dehors, ou plutôt de réaliser entre eux une espèce d'oscillation. »

11. J.-P. Vernant, La mort dans les yeux. Figures de l'Autre en Grèce ancienne, Paris, Hachette Littératures, coll. «Pluriel», 1998, p. 75-82.

12. A. Kiarostami, Abbas Kiarostami. Textes, entretiens, filmographie complète, Paris, Éditions de l'Étoile, p 78-79. Interrogé par Serge Toubiana sur la fin du film, le réalisateur répond: «Des spectateurs me demandent: "Pourquoi dans le film, ne dites-vous pas ce qui arrive au personnage?" À mon tour, je leur retourne la question: "Que pensez-vous personnellement du personnage ?" [...] de manière à ce que les spectateurs puissent les [films inachevés] compléter et y apporter leur propre imaginaire. » Serait-ce ici une manière de convoquer un éphémère interprétatif?

13. R. M. Rilke, "Les élégies de Duino», dans Poésie. Euvres II, Paris, Éditions du Seuil, 1972, p. 368. 